\title{
Emergency lateral canthotomy and cantholysis to relieve intraocular pressure following severe motor vehicle collision
}

\author{
Jason L Elzinga, Herman Bami \\ Faculty Reviewer: Robert Leeper, MD, FRCSC, FACS (Department of Surgery, Division of General Surgery)
}

\section{ABSTRACT}

A young woman displayed a dilated and fixed pupil following a motor vehicle collision with blunt force trauma to face. This was associated with drastically elevated intraocular pressure and proptosis. It was believed that a retrobulbar bleed was causing ocular compartment syndrome, an ischemic condition whereby elevated pressure within the orbit occludes blood flow to the retina and ocular nerves. This is a rare ocular emergency, and must be promptly treated to prevent permanent damage. Ophthalmology was consulted by telephone to confirm the diagnosis and direct treatment. A lateral canthotomy and cantholysis was performed under guidance from Ophthalmology and free open access medical education resources. The trauma team managed to save the eye, with visual acuity remaining near normal. In this report, the mechanism of orbital compartment syndrome is explained, and the lateral canthotomy and cantholysis procedure is outlined. This case highlights the importance of telephone consultation and easily accessible medical education resources in the acute care of rare conditions that may arise following trauma.

\section{CASE PRESENTATION}

A 27-year-old woman was brought to the emergency department by Emergency Medical Services following a severe motor vehicle collision. On arrival, obvious signs of facial trauma were present including a large laceration in the temporal region, open fractures of the right orbital rim, and periorbital ecchymosis. The patient was agitated, with a Glasgow Coma Scale score estimated as 12-13 (E3 V3 M6). Heart rate ranged from 80 to $100 \mathrm{bpm}$, with a blood pressure of roughly 140-150/60-80 mmHg. Respiratory rate was mildly elevated, with good bilateral air entry. Some blood was present in the oropharynx, but there were no signs of airway obstruction.

Due to a high degree of agitation and the need for urgent neuroimaging, the patient was intubated in the emergency department. Intubation was successful following sedation with ketamine and rocuronium. Sedation was maintained with propofol. While sedated, the patient remained normotensive, with oxygen saturation greater than $95 \%$.

On arrival, pupils were initially equal and reactive to light, with a diameter of $4 \mathrm{~mm}$. However, upon completion of the secondary survey, it was noticed that the right pupil had become dilated to $10 \mathrm{~mm}$ and was unresponsive to light. The eye appeared proptot- ic. It was deemed that the patient's previous level of consciousness and active agitation were inconsistent with increased intracranial pressure as an isolated etiology. Uncal herniation with oculomotor nerve (CN III) palsy seemed unlikely to have occurred in such a rapidly progressive fashion.

On further examination, the eye felt firm with intraocular pressures of $12 \mathrm{mmHg}$ (normal) in the left eye and $>50 \mathrm{mmHg}$ in the right eye, as measured using a Tono-Pen. This elevation in intraocular pressure was potentially indicative of a retrobulbar hematoma. Paired with the unresponsive pupil, this suggested that ocular compartment syndrome (OCS) may have been causing ischemic damage to the retina and to nerves supplying the eye. It was believed that a lateral canthotomy and cantholysis should be performed to relieve intraocular and retrobulbar pressure. Ophthalmology was consulted and agreed with the assessment, but were unable to directly assist due to their location off site.

Due to the rarity of this condition, experience performing a lateral canthotomy and cantholysis is limited among emergency providers. A combination of advice over the telephone from Ophthalmology, and a review of a free, open access medical education video about the procedure was utilized to prepare for and perform this rare but emergently required procedure. The trauma surgeon on call performed an emergency lateral canthotomy and cantholysis to relieve pressure and salvage the eye.

\section{CASE OUTCOME}

Following the lateral canthotomy and cantholysis, intraocular pressure returned to normal, between $5-12 \mathrm{mmHg}$ as assessed by Tono-Pen in the trauma bay. The patient was then sent for computed tomography (CT) scans to further assess the extent of her injuries (Figure 1). A right temporal lobe bleed was identified as the cause of intracranial hemorrhage and managed surgically. Following the management of the bleed, facial and ocular reconstruction were also performed by the surgical team in a staged fashion. The patient recovered very well following surgery, and was discharged from the intensive care unit within two weeks. The team managed to save the eye and current visual acuity has been documented as near normal in the affected eye.

\section{DISCUSSION}

Motor vehicle collisions have the potential to cause a wide variety of acute injuries including traumatic brain injury. ${ }^{1}$ With evidence of head trauma, non-reactive pupils have long been observed as a strong predictor of both neurological injury and mortality. ${ }^{2-4}$ 


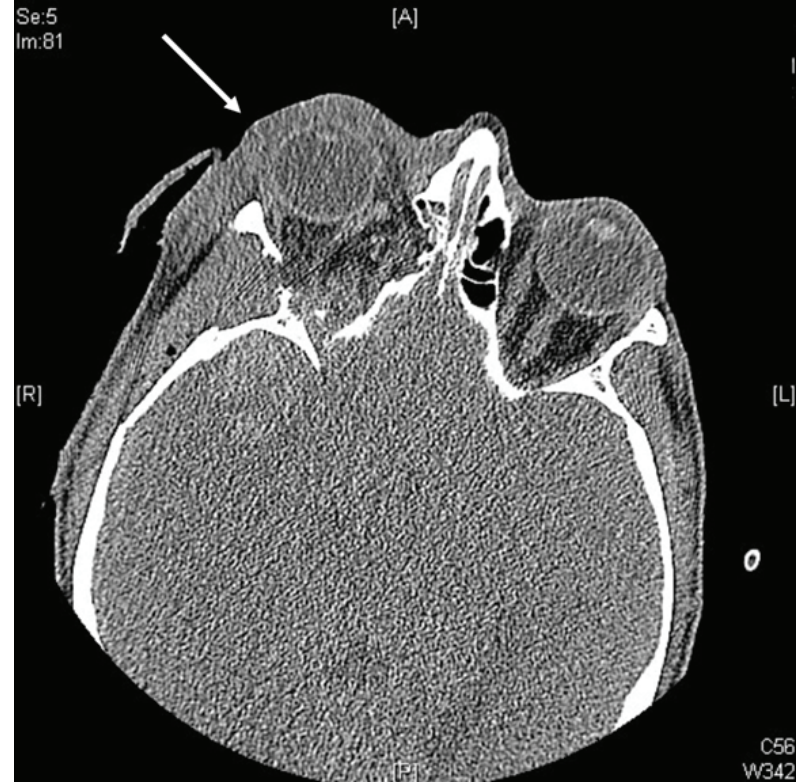

Figure 1. Patient's CT scan following a lateral canthotomy and cantholysis to relieve intraocular pressure. Anterior displacement of the globe can be seen, along with a retrobulbar hematoma associated with multiple fractures to the right orbit.

For this reason, the pupils are routinely assessed as part of the standard ATLS primary survey. A new onset fixed and dilated pupil following head trauma can be caused by increased intracranial pressure in a number of potentially serious neurological conditions including: subdural hematoma, contusion, diffuse brain edema, and epidural hematoma. ${ }^{5}$ However, intracranial pressures significant enough to cause uncal herniation and CN III palsy typically present with additional neurological sequelae.

Traditionally, severe head injury is defined as a patient with a GCS of 8 or less. ${ }^{6}$ Although many neurological signs could not be assessed once the patient was sedated, the patient had been GCS 12-13 and active moments before the dilated pupil was noticed. This made it unlikely that intracranial pressure was solely responsible for the unresponsive pupil. Other ocular findings associated with the unresponsive pupil, including proptosis, a palpably firm eye, periorbital ecchymosis, and intraocular pressure $>50 \mathrm{mmHg}$ were consistent with retrobulbar hematoma as a likely etiology.7

Retrobulbar hemorrhage or hematoma can occur following facial trauma when the vascular supply contained within the orbit, posterior to the eye, is damaged. This cone-shaped space is comprised of bone on all sides, except anteriorly, where it is enclosed by the orbital septum. ${ }^{8}$ Thus, the accumulation of blood within an enclosed, non-compliant space inevitably leads to increased intraocular and orbital pressure. This pressure can impair both blood supply to the retina and the vasa nervorum of CN II and CN III, and is referred to as ocular compartment syndrome (OCS). ${ }^{9}$ OCS requiring urgent surgical management occurs in less than $2 \%$ of all orbital traumas, and therefore is extremely uncommon in most trauma cases. ${ }^{8}$ Ischemic injuries can occur as pressure exceeds the systolic blood pressure in the region and blood supply is impaired.
Animal models have suggested that irreversible damage can occur after only 100 minutes of ischemia. ${ }^{10}$ For this reason, OCS is viewed as a true ophthalmologic emergency, and lateral canthotomy and cantholysis is recommended immediately if intraocular pressure exceeds 40 mmHg. ${ }^{11}$

A lateral canthotomy exposes the lateral canthal tendon, which attaches the superior and inferior tarsal plates of the eyelids to the lateral orbital rim. This then allows for inferior and/or superior aspects of the tendon to be transected (cantholysis). Lateral canthotomy and cantholysis is a procedure designed to rapidly reduce intraocular and orbital pressures by allowing greater anterior displacement of the globe, effectively expanding the volume of the retrobulbar space..$^{12}$ In cadaveric models, this procedure has been shown to decrease orbital and intraocular pressure by an average of $56 \mathrm{mmHg}$ or $53 \%{ }^{13}$ This significant reduction in pressure restores blood flow to the eye and prevents permanent damage.

As orbital compartment syndrome following facial trauma is extremely rare in most emergency departments, experience with recognizing and treating the condition may be limited. A recent report suggested that graduates from an emergency medicine residency program felt that the lateral canthotomy procedure was not important to their practice because of its extremely infrequent use. ${ }^{14}$ This case demonstrates not only the importance of having the skills to recognize and react to rare emergency conditions, but also the potential utility of telephone consultation and electronic medical resources when specialist consultation is not readily available. In this case, a telephone consultation with Ophthalmology was used to confirm the diagnosis and direct treatment, since Ophthalmology was located off site. Data suggests that accessibility of ophthalmology consultation may be limited in emergency departments, especially in rural settings. ${ }^{15}$ Both traditional telephone consultations and newer telemedicine technology may be beneficial in rare ocular emergencies, such as this case. The use of free online medical resources, commonly referred to as free open access medical education (FOAM), also contributed to successful treatment of this rare emergency condition. Evidence suggests that FOAM resources are increasingly being utilized by emergency medical staff to provide optimal patient care. ${ }^{16}$ As of 2012 , there were 183 websites that provided free training and resources for use in emergency medicine and critical care. ${ }^{17}$ Both telemedicine and FOAM resources may have additional utility for managing rare disease entities in an emergency setting, due to the limited familiarity of physicians with these conditions and inherent time constraints.

\section{CONCLUSION}

This report presents a rare case of retrobulbar hematoma and ocular compartment syndrome following blunt facial trauma during a motor vehicle collision. Although the patient's pupils appeared normal on initial presentation, ocular compartment syndrome was suspected after an unresponsive, dilated pupil was noticed and deemed inconsistent with an intracranial pressure etiology. Drastically increased intraocular pressure caused by the accumulation of blood in the orbit threatened permanent damage 
to the eye. A lateral canthotomy and cantholysis was performed to rapidly relieve intraocular and orbital pressure, saving the eye from permanent injury. This case demonstrates not only the importance of considering rare entities in trauma situations, but also the benefit of easily accessible resources when surgeons are required to promptly perform uncommon procedures. Together, the use of a telephone consultation and free open access medical education resources helped to confirm both the diagnosis and management plan. This contributed to a successful procedure, and a positive outcome for the patient.

\section{REFERENCES}

1. Hillary FG, Schatz P, Moelter ST, Lowry JB, Ricker JH, Chute DL. Motor vehicle collision factors influence severity and type of TBI. Brain Inj. 2002 Aug;16(8):729-41.

2. Braakman R, Gelpke GJ, Habbema JDF, Maas AIR, Minderhoud JM. Sytematic selection of prognostic features in patients with severe head injury. Neurosurgery. 1980 Apr;6(4):362-70.

3. Signorini DF, Andrews PJ, Jones PA, Wardlaw JM, Miller JD. Predicting survival using simple clinical variables: a case study in traumatic brain injury. J Neurol Nerurosurg Psychiatry. 1999 Jan;66(1):20-5.

4. Petridis AK, Doukas A, Barth H, Mehdorn M. Outcome of craniocerebral gunshot injuries in the civilian population. Prognostic factors and treatment options. Cent Eur Neurosurg. 2011 Feb;72(1):5-14.

5. Clusmann H, Schaller C, Schramm J. Fixed and dilated pupils after trauma, stroke, and previous intracranial surgery: management and outcome. J Neurol Neurosurg Psychiatry. 2001 Aug;71(2):175-81.

6. Foulkes MA, Eisenberg HM, Jane JA, Marmarou A, Marshall LF. The traumatic coma data bank: design, methods, and baseline characteristics. J Neurogurg. 1991 Nov;75:S8-13.

7. Bailey WK, Kuo PC, Evans LS. Diagnosis and treatment of retrobulbar hemorrhage. J Oral Maxillfac Surg. 1993 Jul;51(7):780-82.

8. Fattahi T, Brewer K, Retana A, Ogledzki M. Incidence of retrobulbar hemorrhage in the emergency department. J Oral Maxillofac Surg. 2014 Dec;72(12):2500-2502.

9. Lima V, Burt B, Leibovitch I, Prabhakaran V, Goldberg RA, Selva D. Orbital compartment syndrome: the ophthalmic surgical ermergency. Surv Ophthalmol. 2009 Jul-Aug;54(4):441-9.

10. Hayreh SS, Zimmerman MB, Kimura A, Sanon A. Central Retinal artery occlusion. Retinal survival time. Exp Eye Res. 2004 Mar;78(3):72336.

11. Adams JG. Emergency medicine: clinical essentials. Pennsylvania: Elsevier Incorporated; 2013. 799 p.

12. Ballard SR, Enzenauer RW, O’Donnell T, Fleming JC, Risk G, Waite AN. Emergency lateral canthotomy and cantholysis: a simple procedure to preserve vision from sight threatening orbital hemorrhage. $\mathrm{J}$ Spec Oper Med. 2009 Summer;9(3):26-32.

13. Oester AE, Fowler BT, Fleming JC. Inferior orbital septum release compared to lateral canthotomy and cantholysis in the management of orbital compartment syndrome. Ophthal Plast Reconstr Surg. 2012 Jan;28(1):40-43.

14. Druck J, Valley MA, Lowenstein SR. Procedural skills training during emergency medicine residency: are we teaching the right things? West J Emerg Med. 2009 Aug;10(3):152-56.

15. Wedekind L, Sainani K, Pershing S. Supply and perceived demand for teleophthalmology in triage and consultations in California emergency departments. JAMA Ophthalmol. 2016 May; 134(5):527-42.
16. Thoma B, Joshi N, Truegar NS, Chan TM, Lin M. Five strategies to effectively use online resources in emergency medicine. Ann Emerg Med. 2014 Oct;64(4):392-5.

17. Cadogan M, Thoma B, Chan TM, Lin M. Free open access meducation (FOAM): the rise of emergency medicine and critical care blogs and podcasts (2002-2013). Emerg Med J. 2014 Oct;31(el):76-7. 